LETTER TO JMG

\title{
Common apolipoprotein E polymorphisms and risk of clinical malaria in the Gambia
}

\author{
C Aucan, A J Walley, A V S Hill
}

J Med Genet 2004;41:21-24

\begin{abstract}
A polipoprotein $\mathrm{E}(\mathrm{apoE})$ is a protein involved in the transport and metabolism of plasma cholesterol and triglycerides. The apolipoprotein E gene (APOE), located at chromosome 19q13.2, has three major alleles called $\varepsilon 2, \varepsilon 3$, and $\varepsilon 4$, defined by two single nucleotide polymorphisms (SNP) located in exon 4 at positions 3937 (T/C) and $4075(\mathrm{C} / \mathrm{T})$. The corresponding apoE isoforms differ at amino acid positions 112 (Cys for apoE2 and apoE3, Arg for apoE4) and 158 (Arg for apoE3 and apoE4, Cys for apoE2), and have been shown to have different functional and biochemical properties. ${ }^{1-4}$ APOE polymorphisms have been studied in relation to several genetic diseases and disorders. The APOE $\varepsilon 4$ allele has been associated with an increased risk of Alzheimer's disease, coronary heart disease, and death after myocardial infarction..$^{5-7}$ Conversely, the APOE $\varepsilon 2$ allele was found to have a protective effect on the occurrence of Alzheimer's disease. However, in other studies, APOE $\varepsilon 2$ was associated with an increased risk of cardiovascular diseases and with some blood lipid abnormalities. ${ }^{89}$ It has been reported recently that APOE $\varepsilon 2$ homozygote children from Ghana became infected with Plasmodium falciparum at an earlier age than those carrying other APOE genotypes. ${ }^{10}$

In this study, performed in the Gambia, the APOE $\varepsilon 2, \varepsilon 3$, and $\varepsilon 4$ allele distributions were analysed in children with mild (338 cases) or severe (530 cases) malaria, and in individually matched control children (560 subjects). Additionally, the APOE Thl/E47 polymorphism located in the APOE promoter region ${ }^{11}$ was studied in a subset of children consisting of 183 severe malaria cases and 179 controls.
\end{abstract}

\section{MATERIALS AND METHODS}

\section{Study subjects}

Between August 1989 and September 1990, 1428 children aged from 1 to 10 years were enrolled at the Royal Victoria Hospital of Banjul and at the Medical Research Council Hospital of Fajara, in the Gambia. Malaria was diagnosed if a patient with an appropriate clinical picture had parasitaemia $>2500 / \mu \mathrm{l}$, and relevant laboratory investigations did not suggest other diagnoses. Cerebral malaria was defined by a Blantyre coma score of more than 3 (persisting for more than 30 minutes after effective treatment of hypoglycaemia or convulsions) or repeated prolonged seizure (for more than 30 minutes) in a child with $P$ falciparum parasitaemia and no other apparent cause of fits or coma. Severe malaria anaemia was defined as a haemoglobin level of less than $50 \mathrm{~g} / \mathrm{l}$ on admission in a child with parasitaemia. Children with mild malaria had an uncomplicated febrile illness with $P$. falciparum parasitaemia and no other apparent cause of fever.

The group of severe malaria patients was matched to two control groups of children for age and area of residence. The mild controls were recruited at both hospitals and health centres in the study area. These children had mild, mostly infective, illnesses that did not require hospital admission and they did not have malarial parasites in their blood on microscopy. Severely infected controls were inpatients at the

\section{Key points}

- Apolipoprotein $E(A p o E)$ is involved in the transport of plasma cholesterol and triglycerides. ApoE polymorphisms have been associated with blood lipid abnormalities and with an increased risk of Alzheimer's disease or cardiovascular disease, in many studies. Recently, the APOE $\varepsilon 2$ allele has been associated with an increased risk of early infection by Plasmodium falciparum in Ghanaian children.

- In this study, APOE $\varepsilon 2, \varepsilon 3, \varepsilon 4$, and APOE Th1/E47 polymorphic markers were genotyped for 1428 children in a Gambian case control study, to evaluate the effect of APOE polymorphisms on the risks of mild and severe malaria.

- In this Gambian sample, APOE allele frequencies were $12.8 \%, 64.4 \%$, and $22.8 \%$ for $\varepsilon 2, \varepsilon 3$, and $\varepsilon 4$, respectively. No differences in frequencies were observed between the controls and either the mild malaria cases or the severe malaria cases. Moreover, no differences were observed between the mild malaria cases and the severe malaria cases. However, in a particular subgroup of children developing both cerebral malaria and severe malarial anaemia, the APOE $\varepsilon 3 / \varepsilon 4$ genotype was found to be more frequent $(42.9 \%)$ than in the controls $(24.8 \%)$ $\left(2 \times 2 \chi^{2}=7.57 ; p=0.006\right)$ or in the mild malaria cases $(27.2 \%)\left(2 \times 2 \chi^{2}=5.06 ; p=0.024\right)$.

- Our results suggest that APOE polymorphisms do not influence protection against cerebral malaria and severe malarial anaemia, but could possibly play a role in protection against a particularly severe form of severe malaria.

two hospitals, with a large range of other acute, mainly infective, illnesses but without evidence of current or recent malaria infection.

The ethnic composition of the population in this area is mixed: Mandinka (42\%), Jola (14\%), Wolof (14\%), Fula $(12 \%)$, and several less common ethnic groups. The children from the different groups were not matched for ethnic group; instead, analyses stratified with this variable were carried out. Details of this case control study have been described previously. ${ }^{12}$

The study had the approval of the Gambian Government and of the MRC Joint Ethical Committee, and consent was obtained from the parent or guardian of each child entered.

\section{Apolipoprotein E genotyping}

Base positions mentioned in this paper refer to the APOE reference sequence GenBank AF261279. Three APOE SNP 
markers, located at positions 832 (APOE-Thl/E47), 3937 (apoE+112), and 4075 (apoE+158), were studied by ligation detection reaction (LDR).$^{13}$ For the APOE-Thl/E47 (G/T) SNP, PCR was performed in $15 \mu \mathrm{l}$ of reaction mixture containing: $50 \mathrm{ng}$ genomic DNA; $10 \mathrm{mM}$ Tris- $\mathrm{HCl} ; 50 \mathrm{mM} \mathrm{KCl}$ (pH 8.3,GeneAmp 10X Buffer II, Perkin Elmer, Beaconsfield, UK); $1.0 \mathrm{mM} \mathrm{MgCl} ; 80 \mu \mathrm{M}$ of each dNTP; $1.0 \mathrm{U}$ AmpliTaq Gold DNA polymerase; and $0.33 \mu \mathrm{M}$ each of forward (5'-TACTTTCTTTCTGGGATCCAGG-3') and reverse (5'-ACTCAAGGATCCCAGACTTG-3') primers. ${ }^{11}$ For the APOE 3937 (T/C) and 4075 (C/T) SNPs, a single PCR was performed in $20 \mu \mathrm{l}$ of reaction mixture containing: $50 \mathrm{ng}$ of genomic DNA; $10 \mathrm{mM}$ Tris-HCl; $50 \mathrm{mM} \mathrm{KCl} \mathrm{(} \mathrm{pH} \mathrm{8.3);} 1.5 \mathrm{mM}$ $\mathrm{MgCl}_{2} ; 50 \mu \mathrm{M}$ of each dNTP; $1.0 \mathrm{U}$ AmpliTaq Gold DNA polymerase; and $0.25 \mu \mathrm{M}$ each of forward (5'-AGATGC GGGCACGGCTGTTCAAGGA-3') and reverse (5'-CCCTCGC GAGCCCCGGCCTGGTACAC-3') primers. ${ }^{14}$ PCR amplification reactions were performed using an MJ Research tetrad thermal cycler as follows: $94^{\circ} \mathrm{C}$ for 14 minutes; 39 cycles of $94^{\circ} \mathrm{C}$ for 60 seconds; $60^{\circ} \mathrm{C}$ (APOE-Thl/E47) or $67^{\circ} \mathrm{C}$ (APOE 3937 and 4075 ) for 60 seconds; $72^{\circ} \mathrm{C}$ for 60 seconds (APOEThl/E47) or 90 seconds (APOE 3937 and 4075); and finally $72^{\circ} \mathrm{C}$ for 7 minutes.

The SNP genotyping was carried out using the LDR method..$^{13}$ For each SNP, the allele specific oligonucleotide probe pairs were distinguished by different fluorescent labels (FAM/HEX) and by their lengths. The common probes were phosphorylated at their $5^{\prime}$ extremities (P). LDR reactions were performed as previously described, ${ }^{15}$ and LDR products were analysed using an ABI 3700 (Applied Biosystems, Warrington, UK). The following probes were used for APOEThl/E47: APOE-Thl/E47-G 5' -(FAM)-AAAAAAAACCCAGAA TGGAGGAGGGTGTCTGG-3', APOE-Thl/E47-T 5'-(HEX)-AA AAAAAACCCCAGAATGGAGGAGGGTGTCTGT-3', and APOEThl/E47-common 5'-(P)-ATTACTGGGCGAGGTGTCCTCCCTT CAAAAAATAAAAAAAAA-3'. For APOE 3937 we used: APOE 3937-C 5'-(FAM)-AAAAAAAAAGCGCGGACATGGAGGACGT GC-3', APOE 3937-T 5'-(HEX)-AAAAAAAAAGGCGCGGAC ATGGAGGACGTGT-3', and APOE 3937-common 5'-(P)-GC GGCCGCCTGGTGCAGTACCAAAAAA-3'. For APOE 4075 we used: APOE 4075-C 5'-(FAM)-AAAAAAAAAAACGATGCCG ATGACCTGCAGAAGC-3', APOE 4075-T 5'-(HEX)-AAAAA AAAAAAGCGATGCCGATGACCTGCAGAAGT-3', and APOE 4075-common 5' -(P)-GCCTGGCAGTGTACCAGGCCGGAAAA AAAATAAA- $3^{\prime}$. Non-APOE sequence nucleotides were added to the LDR probes to increase the size of the LDR products. The APOE $\varepsilon 2$ allele was defined by a T at position 3937 and a $\mathrm{T}$ at position 4075 . The APOE $\varepsilon 3$ allele was defined by a $\mathrm{T}$ at position 3937 and a C at position 4075. The APOE $\varepsilon 4$ allele was defined by a $C$ at position 3937 and a $C$ at position 4075 . The APOE $\varepsilon 2 / \varepsilon 2, \varepsilon 2 / \varepsilon 3, \varepsilon 2 / \varepsilon 4, \varepsilon 3 / \varepsilon 3, \varepsilon 3 / \varepsilon 4$, and $\varepsilon 4 / \varepsilon 4$ genotypes were defined according to individuals' APOE 3937 and APOE 4075 genotypes (for more information see http://www. well.ox.ac.uk/hill/Malaria/APOEgenotyping.htm). Allele frequencies were then calculated using genotype data.

\section{Statistical analyses}

Allele and genotype frequencies were compared between children with malaria and the control children, using the Pearson's $\chi^{2}$ test. Logistic regression analysis was then performed to take into account the effect of confounding factors such as age, gender, ethnicity, and household location. ${ }^{16}$ Statistical analyses were carried out with SPSS version 11 (SPSS Inc, USA) and EpiInfo version 1.1.2 (CDC, USA).

\section{RESULTS}

Three SNPs located either in the promoter region (APOE Thl/ E47) or in exon 4 (APOE 3937 and 4075) of the APOE gene were genotyped. APOE 3937 and 4075 markers were typed in
530 severe malaria cases, 338 mild malaria cases, and 560 individually matched control children. These genotypes were used to define individuals' $\operatorname{APOE} \varepsilon 2, \varepsilon 3$, and $\varepsilon 4$ alleles as described. No differences were observed between controls with mild non-malaria diseases (247 patients) and controls with severe non-malaria diseases (313 patients) for allele frequency and genotype distribution (data not shown). Consequently, these control subjects were grouped as a single control group for subsequent analyses.

The APOE $\varepsilon 2(12.8 \%), \varepsilon 3(64.4 \%)$, and $\varepsilon 4(22.8 \%)$ allele frequencies in this Gambian sample were found to be closer to those reported in Ghanaian samples $(14.5 \%, 61.4 \%$, and $24.1 \%$, respectively, for $\varepsilon 2, \varepsilon 3$, and $\varepsilon 4$ ) than others reported in African or non-African populations. ${ }^{10}{ }^{17}$ As shown in table 1(A), APOE allele frequencies were found to be similar in the control group and in children with severe and with mild malaria . Furthermore, no differences were observed between the different severe malaria subgroups and either the controls or the mild malaria cases (table $1(\mathrm{~A})$ ). APOE genotypes also were compared between these different study groups. Although the APOE $\varepsilon 3 / \varepsilon 4$ genotype was apparently more common in severe malaria $(29.4 \%)$ and cerebral malaria $(29.0 \%)$ cases than in the mild malaria cases $(27.2 \%)$ or in the controls $(24.8 \%)$, no statistically significant differences were observed between these study groups for any APOE genotype. The APOE genotype distribution was, however, found to be significantly different between the control group and children developing both cerebral malaria and severe malarial anaemia $\left(\chi^{2}=11.42, \mathrm{p}=0.044\right)$. In this particular subgroup of children, the APOE $\varepsilon 3 / \varepsilon 4$ genotype was found to be more frequent $(42.9 \%)$ than in the controls $(24.8 \%)$ $\left(2 \times 2 \chi^{2}=7.57, \mathrm{p}=0.006\right)$ and the mild malaria cases $(27.2 \%)\left(2 \times 2 \chi^{2}=5.06, p=0.024\right)$ (table $\left.1(B)\right)$. These results were confirmed by binary logistic regression where additional factors, such as ethnicity, household location, gender, and age, were simultaneously included in the analyses (data not shown).

The APOE Thl/E47 SNP was genotyped in a subset of 183 severe malaria cases and 179 control children (table 2 (A)). This polymorphism is located in a consensus sequence of a potential transcriptional (Thl/E47) factor binding site, and the APOE Thl/E47-T allele was previously reported to be associated with an increased risk of Alzheimer's disease. ${ }^{1{ }^{11}}{ }^{18}$ As shown in table 2 (A), the allele frequencies were similar in severe malaria cases and in control children $(75.4 \%$ and $76.0 \%$, respectively, for the $\mathrm{G}$ allele; $\chi^{2}=0.03, \mathrm{p}=0.87$ ). Similarly, as shown in table 2 (B), genotype frequencies were not found to be significantly different between the severe malaria cases and the control children $\left(\chi^{2}=1.95, \mathrm{p}=0.38\right)$. Because of the absence of significant difference between this subset of 183 severe malaria cases and 179 controls, the rest of the Gambian case control samples were not genotyped for this marker.

\section{DISCUSSION}

The results reported here demonstrate that, in this Gambian case control study, genetic polymorphisms within APOE do not significantly influence host resistance or susceptibility to severe malarial anaemia and cerebral malaria, the two most common forms of severe malaria. Surprisingly, when severe malaria cases were subdivided in clinical categories, the APOE $\varepsilon 3 / \varepsilon 4$ genotype was found to be more common in children with both cerebral malaria and severe malarial anaemia $(42.9 \%)$ than in controls $(24.8 \%)$ and mild malaria cases $(27.2 \%)$. However, these results must been considered with caution because they are based on a relatively small number of cases (49) and could be due simply to chance, or to a biased distribution of the APOE genotype in this particular sample. Indeed, when a correction factor is applied 
Table 1 Distribution of APOE $\varepsilon 2, \varepsilon 3$, and $\varepsilon 4$ alleles in the Gambia

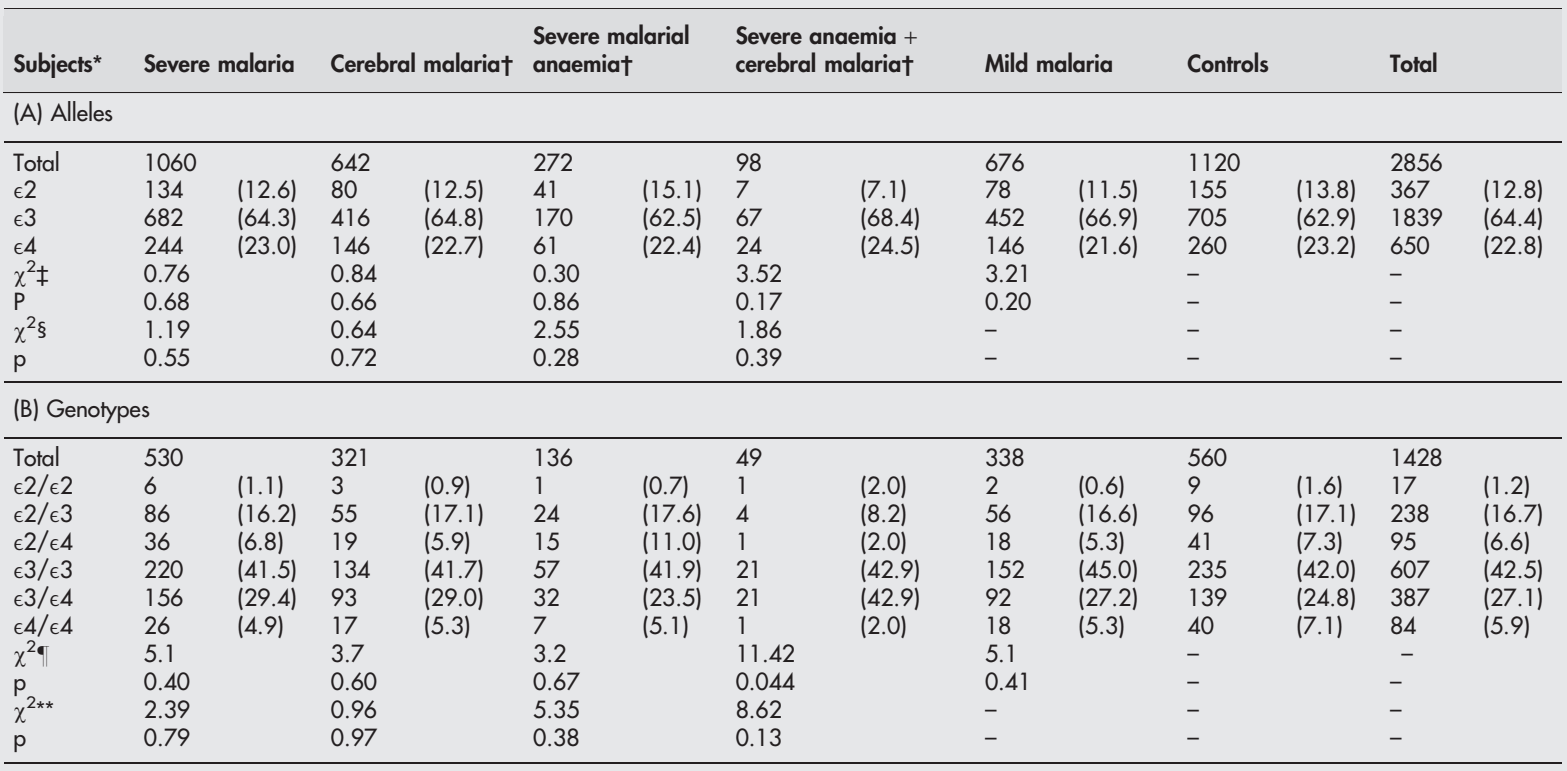

*Percentages within each disease or control group are given in parentheses.

†Children (24) with severe hypoglycaemia only were excluded from this analysis.

$\ddagger 3 \times 2 \chi^{2}$ test ( $\left.d f=2\right)$ for comparison with the control group.

$\S 3 \times 2 \chi^{2}$ test $(\mathrm{df}=2$ ) for comparison with the mild malaria group.

$-6 \times 2 \chi^{2}$ test ( $\mathrm{df}=5$ ) for comparison with the control group.

${ }^{* *} 6 \times 2 \chi^{2}$ test $(\mathrm{df}=5)$ for comparison with the mild malaria group.

for the number of clinical groups (4) compared with controls in this study, this finding loses statistical significance. These results apparently contrast with a recent report from Ghana. ${ }^{10}$ However, different and hence complementary phenotypes were studied in the Gambia (mild and severe clinical malaria) and in Ghana, where children with the APOE $\varepsilon 2 /$ $\varepsilon 2$ genotype were found to be infected with $P$ falciparum sooner than other children. ${ }^{10}$ This difference observed between children with different APOE genotypes could be explained by the fact that the different APOE isoforms have been shown to have different physiological properties and, in particular, have been shown to have different affinities for some of their receptors such as LRP and apoER2. ${ }^{34}$ Moreover, APOE receptors have been shown to bind to the

Table 2 Distribution of APOE Th1/E47 polymorphism in the Gambia

\begin{tabular}{lllllll}
\hline Subjects* & \multicolumn{2}{l}{ Severe malaria } & \multicolumn{2}{l}{ Controls } & Total & \\
\hline (A) Alleles & & & & & & \\
\hline Total & 366 & & 358 & & 724 & \\
$\mathrm{G}$ & 276 & $(75.4)$ & 272 & $(76.0)$ & 548 & $(75.7)$ \\
$\mathrm{T}$ & 90 & $(24.6)$ & 86 & $(24.0)$ & 176 & $(24.3)$ \\
$\chi^{2} \dagger$ & 0.03 & & - & & - & \\
$\mathrm{P}$ & 0.87 & & - & & - & \\
\hline (B) Genotypes & & & & & & \\
\hline Total & 183 & & 179 & & 362 & $(56.3)$ \\
$\mathrm{G} / \mathrm{G}$ & 100 & $(54.6)$ & 104 & $(58.1)$ & 204 & \\
$\mathrm{G} / \mathrm{T}$ & 76 & $(41.5)$ & 64 & $(35.8)$ & 140 & $(38.7)$ \\
$\mathrm{T} / \mathrm{T}$ & 7 & $(3.8)$ & 11 & $(6.1)$ & 18 & $(5.0)$ \\
$\chi^{2} \ddagger$ & 1.95 & & - & & - & \\
$\mathrm{P}$ & 0.38 & & - & & - & \\
\hline
\end{tabular}

*Percentages within each disease or control group are given in parentheses.

$+2 \times 2 \chi^{2}$ test ( $\mathrm{df}=1$ ) for comparison with the control group. $\ddagger 3 \times 2 \chi^{2}$ test $(\mathrm{df}=2)$ for comparison with the control group. circumsporozoite protein (CSP) from plasmodium sporozoites. In mice models, sporozoites were shown to be less able to invade hepatic cells from LDLR knockout mice. ${ }^{19}$ Therefore, it is possible that a particular apoE isoform, such as apoE2, is less efficient than the other isoforms in competing with the sporozoites for binding to their receptor on hepatic cells, and hence influence host resistance to malaria infection. In Ghana, APOE polymorphisms were not found to influence individual blood infection levels. ${ }^{10}$ In our study, the APOE $\varepsilon 3 /$ $\varepsilon 4$ genotype was found to be more frequent in a small group of children (49) developing cerebral malaria and severe malarial anaemia simultaneously. This genotype was however not found to be significantly more frequent in children developing cerebral malaria only or severe malarial anaemia only, suggesting that APOE genotypes could have a role in the genetic control of this particular form of severe malaria; alternatively, this may simply be a chance result.

These results suggest that APOE polymorphisms do not influence protection against cerebral malaria and severe malarial anaemia, but could have a role in protection against the early stages of the infection and possibly against a particularly severe form of severe malaria (cerebral malaria plus severe malarial anaemia). Further studies are required to assess the role of APOE polymorphisms in the risk of severe malaria in other populations.

\section{ACKNOWLEDGEMENTS}

We are grateful to the people of the Gambia for their cooperation. We thank C Allsopp, D Kwiatkowski, D Brewster, and N Anstey for their contributions to the design and execution of this case control study.

\section{Authors' affiliations}

C Aucan, A J Walley, A V S Hill, The Wellcome Trust Centre for Human Genetics, Roosevelt Drive, Oxford OX3 7BN, UK

The study was funded by the MRC and the Wellcome Trust.

A V S Hill is a Wellcome Trust Principal Research Fellow. 
Correspondence to: C Aucan, The Wellcome Trust Centre for Human Genetics, Roosevelt Drive, Oxford OX3 7BN, UK; aucan@well.ox.ac.uk

Received 2 July 2003

Accepted 16 September 2003

\section{DATA ACCESS}

GenBank: AF261279; OMIM: 107741

\section{REFERENCES}

1 Weisgraber KH. Apolipoprotein E: structure-function relationships. Adv Protein Chem 1994;45:249-302.

2 Gregg RE, Brewer Jr HB. The role of apolipoprotein E and lipoprotein receptors in modulating the in vivo metabolism of apolipoprotein B-containing lipoproteins in humans. Clin Chem 1988;34(8B):B28-32.

3 Boerwinkle $E$, Utermann $G$. Simultaneous effects of the apolipoprotein $E$ polymorphism on apolipoprotein $E$, apolipoprotein $B$, and cholesterol metabolism. Am J Hum Genet 1988;42(1):104-12.

4 Mamotte CD, Sturm M, Foo J, van Bockxmeer FM, Taylor RR. Comparison of the LDL-receptor binding of VLDL and LDL from apoE4 and apoE3 homozygotes. Am J Physiol 1999;276(3):E553-7.

5 Laws SM, Hone E, Gandy S, Martins RN. Expanding the association between the APOE gene and the risk of Alzheimer's disease: possible roles for APOE promoter polymorphisms and alterations in APOE transcription. J Neurochem 2003;84(6): 1215-36.

6 Mahley RW, Rall Jr SC. Apolipoprotein E: far more than a lipid transport protein. Annu Rev Genomics Hum Genet 2000;1:507-37.

7 Gerdes LU, Gerdes C, Kervinen K, Savolainen M, Klausen IC, Hansen PS, et al. The apolipoprotein epsilon4 allele determines prognosis and the effect on prognosis of simvastatin in survivors of myocardial infarction: a substudy of the Scandinavian simvastatin survival study. Circulation 2000;101(12):1366-71.

8 Breslow JL, Zannis VI, SanGiacomo TR, Third JL, Tracy T, Glueck CJ. Studies of familial type III hyperlipoproteinemia using as a genetic marker the apoE phenotype E2/2. J Lipid Res 1982;23(8):1224-35.
9 Corder EH, Saunders AM, Risch NJ Strittmatter WJ Schmechel DE Gaskell Jr PC, et al. Protective effect of apolipoprotein E type 2 allele for late onset Alzheimer disease. Nat Genet 1994;7(2):180-4.

10 Wozniak MA, Faragher EB, Todd JA, Koram KA, Riley EM, Itzhaki RF. Does apolipoprotein E polymorphism influence susceptibility to malaria? J Med Genet 2003;40(5):348-51.

11 Lambert JC, Pasquier F, Cottel D, Frigard B, Amouyel P, Chartier-Harlin MC A new polymorphism in the APOE promoter associated with risk of developing Alzheimer's disease. Hum Mol Genet 1998;7(3):533-40.

12 Hill AV, Allsopp CE, Kwiatkowski D, Anstey NM, Twumasi P, Rowe PA, et al. Common West African HLA antigens are associated with protection from severe malaria. Nature 1991;352(6336):595-600.

13 Day DJ, Speiser PW, Schulze E, Bettendorf M, Fitness J, Barany F, et al. Identification of non-amplifying CYP21 genes when using PCR-based diagnosis of 21-hydroxylase deficiency in congenital adrenal hyperplasia (CAH) affected pedigrees. Hum Mol Genet 1996;5(12):2039-48

14 Shcherbak NS. Apolipoprotein E gene polymorphism is not a strong risk factor for diabetic nephropathy and retinopathy in Type I diabetes: casecontrol study. BMC Med Genet 2001;2(1):8.

15 Aucan C, Walley AJ, Hennig BJ, Fitness J, Frodsham A, Zhang L, et al. Interferon-alpha receptor-1 (IFNAR1) variants are associated with protection against cerebral malaria in the Gambia. Genes Immun 2003;4(4):275-82

16 Hosmer DW, Lemeshow S. Applied logistic regression. In: Assessing the fit of the model. New York: Wiley, 1989:135-75.

17 Gerdes LU, Gerdes C, Hansen PS, Klausen IC, Faergeman O, Dyerberg J. The apolipoprotein E polymorphism in Greenland Invit in its global perspective. Hum Genet 1996;98(5):546-50.

18 Lambert JC, Berr C, Pasquier F, Delacourte A, Frigard B, Cottel D, et al. Pronounced impact of Th1/E47cs mutation compared with - 491 AT mutation on neural APOE gene expression and risk of developing Alzheimer's disease. Hum Mol Genet 1998;7(9):1511-16.

19 Shakibaei M, Frevert U. Dual interaction of the malaria circumsporozoite protein with the low density lipoprotein receptor-related protein (LRP) and heparan sulfate proteoglycans. J Exp Med 1996;184(5):1699-711.

\section{$\mathrm{ECHO}$}

\section{DQB 1 alleles affect susceptibility to pulmonary TB in Europeans}

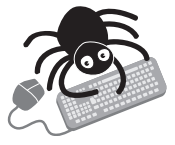

Please visit the Journal of Medical Genetics website [ww.jimedgenet. com] for a link to the full text of this article.
$\mathrm{T}$ he first study of its kind in white Europeans has suggested that the allelic frequency of histocompatibility genes is linked to susceptibility to pulmonary TB.

The frequency of DBQ1 alleles in a white Polish population showed a significant positive association of $\mathrm{DQB1}{ }^{*} 05$ and pulmonary $\mathrm{TB}$, with patients almost three times more likely to have DQB1*05 as controls (odds ratio (OR) 2.84; 95\% confidence interval (CI) 1.57 to 5.15 ). This has been reported in many other ethnic populations.

On the other hand, patients were significantly less likely to have DQBl*02 (OR 0.39; 95\% CI 0.21 to 0.71 ), which has not been noted elsewhere. Other alleles-DQBl*03, DQB1*04, and $\mathrm{DQB1}{ }^{*} 06$ - had similar frequencies in patients and controls.

This preliminary study included 38 unrelated patients with newly identified active pulmonary TB in one hospital and 58 unrelated controls of similar age. Individuals in both groups were excluded, as necessary, to ensure uniform socioeconomic and ethnic background. All had had BCG immunisation. HLA typing was by low resolution PCR with sequence specific primers (PCR-SSP) of blood samples.

The study provides yet more evidence that variation in the HLA system affects response to infection in pulmonary TB. Only 1 in 10 of infected persons will develop the active disease, and genetic differences in immunological defences, and therefore the HLA system, have been implicated. The frequencies of DQB alleles have been studied in Asian and American ethnic groups, but not in Europe, despite an increasing incidence of TB there.

A Thorax 2003;58:890-891. 
Polymorphisms and malaria

\section{Apolipoprotein E polymorphisms and risk of malaria}

\section{A Wozniak, E M Riley, R F Itzhaki}

$\mathrm{H}$ ost genetic factors probably determine both susceptibility to infection and severity of damage by pathogens. A large number of polymorphisms have now been implicated in the onset, progression, and outcome of malaria infection, seeming to influence the ability of the host immune response to control the infection. These include:

- those associated with haemoglobinopathies $^{1}$

- those within the major histocompatibility complex (HMC), including HLA class I and class II and the tumour necrosis factor promoter

- those within genes not associated with HMC, such as ICAM-1, CD36, and possibly the gene for nitric oxide synthase

- probably the gene for apolipoprotein E (APOE), as our recent data suggest, and upon which we comment below. ${ }^{3}$

APOE has three main alleles, types 2 , 3, and 4, resulting in six possible genotypes; it codes for the protein apoE, which is involved in transport of lipids in the blood and the central nervous system.

Finding that APOE- $\varepsilon 2$ homozygous Ghanaian infants were more likely to be infected with the malaria protozoon at a very young age than those with the other genotypes, we predicted that severity of illness after infection might depend to some extent on APOE genotype. ${ }^{3}$ In this issue, the article by Aucan et al offers support for involvement of APOE in the development of severe malaria: people carrying an APOE- $83 /$ \&4 genotype may be more likely than those with the other main genotypes to suffer extremely severe malaria (cerebral malaria and severe anaemia). Our prediction stemmed from previous observations that APOE determines outcome of infection, rather than susceptibility to infection, by three very diverse pathogens:

- herpes simplex virus type l (HSVl) in cold sores $^{45}$ and herpes simplex encephalitis $^{6}$
- hepatitis $C$ virus in liver damage ${ }^{7}$

- HIV in dementia and peripheral neuropathy occurring before the development of AIDS. ${ }^{8}$

These studies followed our previous discovery that HSVl resides in a high proportion of elderly human brains," and that in APOE- 84 carriers this virus confers a strong risk of Alzheimer's disease. $^{45}$ We tentatively suggested ${ }^{5}$ an explanation based on the usage by each of these viruses, ${ }^{710}$ and by the malaria protozoon, ${ }^{3}$ of the same cell entry mediators as those of apoE. These mediators include surface binding sites (heparan sulphate proteoglycans or HSPG) and/or receptors (one of the low density lipoprotein receptor family). Thus, apoE might compete with the pathogens for entry into cells, and any difference in isoform affinity for the binding sites/receptors could affect the extent of competition and hence of pathogen entry, spread, and damage. In fact, apoE isoforms do have different affinities for certain cells ${ }^{11}{ }^{12}$ : with hepatoma cells apoE4 has the least affinity, whereas with fibroblasts there are no differences. ${ }^{11}{ }^{12}$ This might explain why a specific allele is protective in some cases $^{7}$ but harmful, ${ }^{3-68}$ or else neutral, ${ }^{13}$ in others.

Competition between ApoE and the protozoon might be a factor also in very severe malaria. ApoE isoforms may vary in their ability to compete with malaria sporozoites for binding to hepatocytes, thereby affecting parasite load in the liver, the density of forms of the parasite in the blood, and thus the severity of the disease.

Severe malaria is also associated with changes in adhesive properties of infected erythrocytes to endothelial cells and with rosetting, both of which cause obstructions in brain microvasculature; these processes, as well, involve cell surface HSPG. ${ }^{14}$ Thus, as described above, apoE isoforms might affect-via HSPG - the rate or extent of spread of the parasite in erythrocytes and the consequent adhesive changes in these cells. Alternatively, differences in plasma level of apoE and/or lipoproteins that are known to occur in the different genotypes might affect erythrocyte adhesion.

Our discovery that APOE- $\varepsilon 2$ homozygotes are infected at a very early age is not inconsistent with the finding of Aucan et al that APOE- $\varepsilon 3 / \varepsilon 4$ carriers are more likely to suffer extremely severe malaria. Epidemiological studies ${ }^{15}$ suggest that the risk of developing severe malaria is lower in children who experience their first malaria infections very early in life than in those first infected at an older age. Presumably, children infected during infancy (while still protected from clinical malaria and high parasitaemia by innate protective mechanisms and maternal antibodies) develop adaptive immune responses that protect them from severe disease in later life. On the other hand, those infected only later (after the protective mechanisms of infancy have waned) are fully susceptible and at high risk of severe or fatal disease. Thus, the earlier the infection occurs (as in $\varepsilon 2$ homozygotes), the less the likelihood of lifethreatening illness. Indeed, in the study by Aucan et al, APOE- $\varepsilon 2$ carriers were under-represented in the extremely severe malaria (cerebral malaria with severe anaemia) group (7.1\% $v \quad 11.5-$ $13.8 \%$ in the other groups), although the difference does not reach statistical significance.

Whatever the explanation for the results of Aucan et al, our data and theirs add to the extraordinarily diverse repertoire of infective diseases in which APOE determines outcome of, or susceptibility to, infection; and they suggest the possible use of this information for prognostic purposes.

J Med Genet 2004;41:145-146. doi: 10.1136/jmg.2003.014613

\section{Authors' affiliations}

M A Wozniak, R F Itzhaki, Department of Optometry \& Neuroscience, UMIST, Manchester, UK

E M Riley, Department of Infectious and Tropical Diseases, London School of Hygiene and Tropical Medicine, UK

Correspondence to: $\operatorname{Dr} M$ A Wozniak, Molecular Neurobiology Laboratory, Department of Optometry and Neuroscience, UMIST, Manchester M60 1QD, UK : matthew.a.wozniak@umist.ac.uk

\section{REFERENCES}

1 Weatherall DJ, Clegg JB. Genetic variability in response to infection: malaria and after. Genes Immun 2002;3:331-7.

2 Fortin A, Stevenson MM, Gros P. Susceptibility to malaria as a complex trait: big pressure from a tiny creature. Hum Mol Genet 2002; 1 1:2469-78

3 Wozniak MA, Faragher BE, Todd JA, Koram KA, Riley EM, Itzhaki RF. Does apolipoprotein E polymorphism influence susceptibility to malaria? J Med Genet 2003;40:348-51 
4 Lin WR, Graham J, MacGowan SM, Wilcock GK, Itzhaki RF. Alzheimer's disease, herpes virus in brain, apolipoprotein E4 and herpes labialis. Alzheimers Reports 1998;1:173-8.

5 Itzhaki RF, Lin WR, Shang D, Wilcock GK Faragher B, Jamieson GA. Herpes simplex virus type 1 in brain and risk of Alzheimer's disease. Lancet 1997:349:241-4.

6 Lin WR, Wozniak MA, Esiri MM, Klenerman P, Itzhaki RF. Herpes simplex encephalitis: involvement of apolipoprotein E genotype. J Neurol Neurosurg Psychiatry 2001:70:117-19.

7 Wozniak MA, Itzhaki RF, Faragher EB, James MW, Ryder SD, Irving WL. Apolipoprotein E-epsilon 4 protects against severe liver disease caused by hepatitis $C$ virus. Hepatology 2002;36:456-63
8 Corder EH, Robertson K, Lannfelt L, Bogdanovic N, Eggertsen G, Wilkins J, Hall C. HIV-infected subjects with the E4 allele for APOE have excess dementia and peripheral neuropathy. Nat Med 1998;4:1182-4

9 Jamieson GA, Maitland NJ, Wilcock GK, Craske J, Itzhaki RF. Latent herpes simplex virus type 1 in normal and Alzheimer's disease brains. J Med Virol 1991;33:224-7.

10 Itzhaki RF, Wozniak M, Dobson C, Lin WR. ApoE-viral interactions. Nat Med 1998;4:1344.

11 Bohnet K, Pillot T, Visvikis S, Sabolovic N, Siest G. Apolipoprotein (apo) E genotype and apoE concentration determine binding of normal very low density lipoproteins to HepG2 cell surface receptors. J Lipid Res 1996;37:1316-24.
12 Mamotte CD, Sturm M, Foo Jl, van Bockxmeer FM, Taylor RR. Comparison of the $\mathrm{LDL}$-receptor binding of VLDL and LDL from apoE4 and apoE3 homozygotes. Am J Physiol 1999:276:E553-7.

13 Lin WR, Tullo AB, Itzhaki RF. Apolipoprotein E and herpes virus diseases: herpes simplex keratitis. Eur J Hum Genet 1999:7:401-3.

14 Kisilevsky R, Crandall I, Szarek WA, Bhat S Tan C, Boudreau L, Kain KC. Short-chain aliphatic polysulfonates inhibit the entry of Plasmodium into red blood cells. Antimicrob Agents Chemother 2002:46:2619-26.

15 Gupta S, Snow RW, Donnelly CA, Marsh K, Newbold C. Immunity to non-cerebral severe malaria is acquired after one or two infections. Nat Med 1999:5:340-3.

\section{CORRECTION}

doi: 10.1136/jmg.2003.014613corr 1

This commentary should have been published alongside the article "Common apolipoprotein E polymorphisms and risk of clinical malaria in The Gambia" by $\mathrm{C}$ Aucan, AJ Walley, and AVS Hill in the January 2004 issue ( $\mathrm{J}$ Med Genet 2004;41:2124). This error is much regretted and we would like to offer our sincere apologies to the authors involved. 